\title{
Budget Impact Analysis of Eliglustat for the Treatment of Gaucher Disease Type 1 in the United States
}

\author{
Luba Nalysnyk MD, MPH; Rebecca Sugarman, MS; Clifford Cele, BA; \\ Jennifer Uyei, PhD, MPH; and Alexandra Ward, PhD, MRPharms
}

\begin{abstract}
BACKGROUND: Gaucher disease type 1 (GD1) is a rare, genetic, lysosomal storage disease with no cure. Current treatment options include intravenous (IV) enzyme replacement therapy ([ERT]; imiglucerase, velaglucerase alfa, or taliglucerase alfa) or oral substrate reduction therapy ([SRT]; eliglustat or miglustat). The cost to U.S. payers of an IV-administered drug can vary depending on the site of care (i.e., home, outpatient clinic, or hospital setting). Treatment with oral eliglustat may present an opportunity for cost savings.
\end{abstract}

OBJECTIVE: To evaluate the budget impact from a U.S. payer perspective associated with transitioning patients receiving ERTs to the oral SRT eliglustat for the treatment of adults with GD1.

METHODS: A budget impact model estimated the change in pharmaceutical and administration costs resulting from increasing the market share of eliglustat from $12 \%$ (current) to $44 \%$ (new). The market share for eliglustat was drawn equally from existing shares of imiglucerase (40\%) and velaglucerase alfa $(40 \%)$ and assumed to be static over the analysis period. ERT costs were adjusted to account for site of care-based markup and the proportion of patients receiving infusions in each site of care (home, infusion center, or hospital outpatient). Annual ERT costs were calculated assuming a biweekly dose of $47.4 \mathrm{U}$ per $\mathrm{kg}$, a 72-kg patient weight, and 24 infusions per year. The effect of key variables was tested in the sensitivity analyses. All costs are expressed in 2017 U.S. dollars.

RESULTS: In a new plan with 5 million members and 25 GD1 treated patients, increased use of eliglustat resulted in an annual savings of $\$ 1,526,710$ and a total savings of $\$ 4,580,130(13.6 \%)$ over 3 years. The corresponding annual per member per month savings was $\$ 0.025$. This is further illustrated in the sensitivity and scenario analyses where the use of eliglustat was cost saving in all cases. Shifting more patients receiving ERT in the hospital outpatient setting to eliglustat resulted in increased savings. CONCLUSIONS: Based on these analyses, increased use of eliglustat resulted in meaningful cost savings to a payer's overall budget. Cost savings are highest among patients switching from ERT administered in a hospital outpatient setting. The results suggest that cost savings are also likely achievable from initiating patients on oral eliglustat instead of infusion-based therapy from the outset of treatment.

J Manag Care Spec Pharm. 2018;24(10):1002-08

Copyright @ 2018, Academy of Managed Care Pharmacy. All rights reserved.

\section{What is already known about this subject}

Intravenous infusion of enzyme replacement therapy (ERT) imiglucerase is the standard therapy for Gaucher disease type 1 (GD1), but ERT infusions, including velaglucerase and taliglucerase, are expensive, with costs varying significantly depending on patient weight and site of care.

Eliglustat is an orally administered therapy with comparable efficacy to imiglucerase and a favorable safety profile that was approved as a first-line therapy for adults with GDl who are extensive, intermediate, or poor cytochrome P450 2D6 metabolizers.

With oral eliglustat, there are no infusion-associated costs and less variability in price compared with ERT.

\section{What this study adds}

This budget impact study quantifies the economic effect associated with shifting patients from infusion-based therapy to orally administered eliglustat for the treatment of GD1 patients in the United States.

Study results demonstrate that increased use of the orally administered eliglustat instead of the more expensive infusion-based therapy resulted in significant cost savings to third-party payers. Cost savings are also achievable by starting patients on oral eliglustat instead of infusion-based therapy from the outset of treatment.

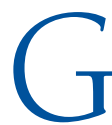
aucher disease (GD) is a rare, genetic lysosomal storage disorder characterized by a loss in enzymatic activity of beta-glucosidase due to mutations in the glucocerebrosidase gene. ${ }^{1,2}$ The prevalence of GD is about 1 to 2 per 100,000 in the general population and is much higher among people of Ashkenazi Jewish descent (an estimated 1 in 850). ${ }^{2}$ Of the 3 main subtypes of GD, Gaucher disease type 1 (GD1) is the most prevalent, accounting for approximately $94 \%$ of all GD diagnoses in the general population. ${ }^{3}$ Impaired activity of beta glucosidase leads to the accumulation of a fatty substance, glucosylceramide, in the viscera (i.e., liver, spleen), bone marrow, and bone, ultimately resulting in tissue and organ damage. Systemic manifestations of the disease when untreated include anemia, thrombocytopenia, hepatosplenomegaly, and skeletal complications such as severe bone pain, avascular necrosis, and joint deformities, or loss in bone mineral density with increased risk of bone fractures. ${ }^{4}$ Among patients diagnosed with GDl in childhood, 35\% experienced growth deficits. ${ }^{5}$ 
Current therapeutic options for GDl in the United States are intravenous (IV) enzyme replacement therapy (ERT) and oral substrate reduction therapy (SRT). ERT augments the defective enzyme with exogenous recombinant beta glucocerebrosidase, while SRT partially inhibits the synthesis of glucosylceramide, thus decreasing its production and accumulation. ${ }^{6}$ Available ERTs include imiglucerase, velaglucerase alfa, and taliglucerase alfa, ${ }^{1}$ and available SRTs include eliglustat (Cerdelga, Sanofi Genzyme) and miglustat. ${ }^{1}$ For many years, imiglucerase has been the standard of care therapy since it was licensed in the early 1990s; it effectively reverses and prevents the manifestations of GDl in the majority of patients (>90\%). ${ }^{6-8}$ Imiglucerase is the first human recombinant enzyme and is produced in Chinese hamster ovary cells.

More recently, 2 other ERTs were approved by the U.S. Food and Drug Administration as alternative products for GD: velaglucerase alfa, produced in human fibroblast cell lines, and taliglucerase alfa, produced in transgenic carrot cells. ${ }^{9}$ However, for some patients, ERT is unsuitable because of an aversion to IV delivery, the lifelong burden of frequent infusions (often every other week), poor venous access, or side effects such as hypersensitivity.

Miglustat, an oral SRT approved in the United States in 2003, is indicated for symptomatic patients with mild to moderate GDl for whom ERT is not a therapeutic option. Due to its frequent side effects such as gastrointestinal problems, tremor, and peripheral neuropathy, miglustat is limited to a second-line therapy.$^{10}$ More recently in 2014, eliglustat, a licensed oral SRT with comparable efficacy to imiglucerase and a favorable safety profile, was approved in the United States as a first-line therapy for adults with GDl who are extensive, intermediate, or poor cytochrome P450 2D6 (CYP2D6) metabolizers. ${ }^{6,11-18}$ CYP2D6 ultra-rapid metabolizers may not achieve adequate blood concentration of eliglustat to achieve therapeutic effect and are, thus, not included as part of the indication. Extensive, intermediate, and poor metabolizer categories encompass greater than $90 \%$ of GDl patients, indicating that most patients are eligible for eliglustat based on their CYP2D6 status. ${ }^{17,18}$

The cost of an IV-administered drug can vary by more than $100 \%$ depending on disease severity and patient weight (as dosage is individualized), as well as where patients go to receive their infusions (i.e., home, outpatient clinic, or hospital outpatient setting). The hospital outpatient setting accounts for a disproportionate percentage of the total spend and is the site of care where the majority of infusions take place. ${ }^{19}$ Efforts have been made by payers with varying success to redirect patients away from hospital infusions where drug markups are highest to less costly settings, particularly the patient's home, not only for GD but for a variety of chemotherapy and nonchemotherapy infusions. ${ }^{20}$ Patients may also prefer infusions at home due to their convenience, easier childcare, no need to travel, and less

\section{TABLE 1 Formulary Market Share Distributions,} Year $1^{\text {a }}$

\begin{tabular}{l|c|c} 
Treatment & $\begin{array}{c}\text { Current Market Shares } \\
\%\end{array}$ & $\begin{array}{c}\text { New Market Shares } \\
\%\end{array}$ \\
\hline Eliglustat & 12 & 44 \\
\hline Miglustat & 4 & 4 \\
\hline Imiglucerase & 40 & 24 \\
\hline Velaglucerase & 40 & 24 \\
\hline Taliglucerase & 4 & 4 \\
\hline
\end{tabular}

aFrom Sanofi Genzyme internal data on file, 2015.

disruption to their family/social life. ${ }^{20,21}$ Drugs administered orally at home incur no administration costs. Thus, the transfer of patients from ERT to oral eliglustat may present cost-saving opportunities for payers.

The objective of this study was to evaluate the budgetary impact from a U.S. payer perspective associated with transitioning patients receiving ERT treatments to the oral SRT eliglustat for the treatment of adults with GDl.

\section{Methods}

\section{Model Overview}

A decision-analytic economic model was developed to investigate the economic impact of a change in the market share of eliglustat to a payer's formulary for the treatment of GDl in adults in the United States. Two market formularies were compared, as shown in Table 1. The first formulary reflected the current market, in which eliglustat represented $12 \%$ of the market share, and the second formulary reflected a hypothetical new market where the share for eliglustat was increased to $44 \%$ and was drawn equally from existing shares of imiglucerase and velaglucerase. In the new market, it was assumed eliglustat did not take shares from miglustat and taliglucerase.

The model framework was developed from the perspective of a third-party payer in the United States. Patient copayment and coinsurance were not considered, as this would introduce significant complexity related to shifting between medical and pharmacy benefits due to changes in care locations and routes of administration, yet have minimal effect on the analysis. The budget assessment compared the cost of a typical regional hospital formulary with current market shares and a new formulary with different market shares. The costs included were pharmaceutical (ERT/SRT) and IV infusion administration for ERT treatments. Neither dispensing costs for medications nor monitoring costs were considered in this analysis, and it was assumed they would not differ substantially between ERT and SRT. The primary outcomes of interest were total annual cost, per member per month (PMPM) cost, and the incremental difference between those outcomes in the current and new formularies. Secondary outcomes included the total cost over a 


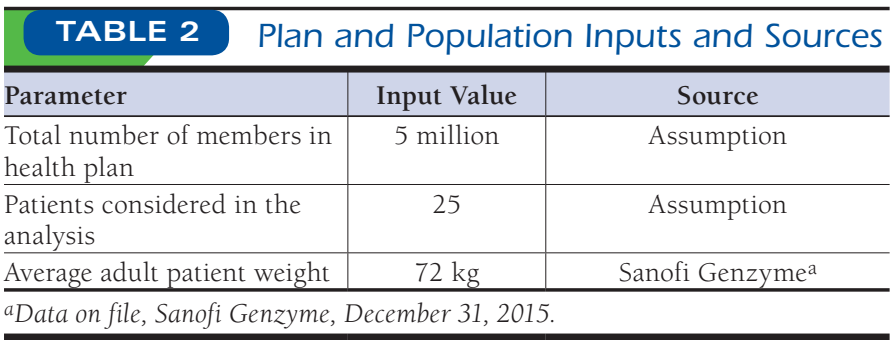

3-year time horizon. The model was developed using Microsoft Excel 2013 (Microsoft, Redmond, WA).

\section{Population}

The population considered in the model consisted of symptomatic adult patients diagnosed with GDl who were eligible for treatment with eliglustat. The estimated number of members in the health plan was 5 million, and the estimated number of patients considered in the analysis was 25 . The average weight per patient was $72 \mathrm{~kg}$ (Table 2).

\section{Model Inputs}

The model inputs for the base-case and sensitivity analyses are shown in Table 3. The 2017 RED BOOK Online values were used to price all ERTs and SRTs. ${ }^{22}$ For ERTs, drug acquisition costs were based on the average dose ( $47.4 \mathrm{U}$ per $\mathrm{kg}$ ), frequency of infusion (24 infusions per year, no discontinuation), and the price per vial of each drug. Dosage (average weight-based dose and infusion frequency) was determined from Sanofi Genzyme internal data on file using real-world ERT dosage of imiglucerase and was assumed to be the same for velaglucerase and taliglucerase (data on file, Sanofi Genzyme, December 31, 2015). The dose per infusion was then used to estimate the minimum number of vials per infusion.

We illustrated a typical case with a drug markup of $20 \%$ when the site of care was at home, a 25\% markup when the site of care was at an outpatient infusion center, and a 100\% markup for care at an outpatient hospital. ${ }^{19,23}$ We assumed that $36 \%$ of patients received their infusions at home, $32 \%$ at an outpatient infusion center, and 32\% at an outpatient hospital. The associated infusion costs at each setting were derived from a claims database analysis (unpublished claims analysis, DataMart Multiplan [IMPACT] database licensed from OPTUM Insight Life Science by Sanofi U.S. Services, 2013). For SRT, the daily dose for eliglustat and miglustat was $200 \mathrm{mg}$ and $300 \mathrm{mg}$, respectively, based on prescribing information, with adherence assumed to be equal to ERT. SRTs have a fixed oral daily dose and are not dependent on weight. Medication possession ratio (MPR) was assumed at $92.3 \%$ for eliglustat and miglustat (equivalent to 24 of the 26 annual infusions, so that the annual compliance for SRTs and ERTs was kept equal in the absence of

\section{TABLE 3 ERT/SRT-Related Inputs and Sources}

\begin{tabular}{|c|c|c|}
\hline Parameter (Dose) ${ }^{a}$ & Pack Size $^{a}$ & Cost per Pack \\
\hline \multicolumn{3}{|l|}{ Drug acquisition costs-ERT } \\
\hline Imiglucerase $(47.4 \mathrm{U} / \mathrm{kg})^{\mathrm{c}}$ & $1 \times 400 \mathrm{U}$ vial & $1,586.00$ \\
\hline Velaglucerase $(47.4 \mathrm{U} / \mathrm{kg})^{\mathrm{c}}$ & $1 \times 400 \mathrm{U}$ vial & $1,377.00$ \\
\hline Taliglucerase $(47.4 \mathrm{U} / \mathrm{kg})^{\mathrm{c}}$ & $1 \times 200 \mathrm{U}$ vial & 779.45 \\
\hline \multicolumn{3}{|l|}{ Drug acquisition costs-SRT } \\
\hline Eliglustat (200 mg daily)c,d & $56 \times 100 \mathrm{mg}$ cap & 23,800 \\
\hline Miglustat (300 mg daily)c,d & $90 \times 100$ mg cap & 26,820 \\
\hline Parameter & Input Value & Source \\
\hline \multicolumn{3}{|l|}{ Administration-ERT } \\
\hline Frequency of infusion ${ }^{e}$ & 24 infusions/year & \\
\hline \multicolumn{3}{|c|}{ Administration cost by site of care-ERT } \\
\hline Home & $\$ 180^{\mathrm{b}}$ & \multirow{3}{*}{$\begin{array}{l}\text { DataMart Multiplan } \\
\text { (IMPACT) database }\end{array}$} \\
\hline Outpatient, infusion center & $\$ 249^{b}$ & \\
\hline Outpatient, hospital & $\$ 369^{\mathrm{b}}$ & \\
\hline \multicolumn{3}{|c|}{$\begin{array}{l}\text { From RED BOOK Online, } 2017.22 \\
\text { All costs are expressed in } 2017 \text { U.S. dollars. } \\
\text { From drug prescribing information. }{ }^{28-32} \\
\text { Medication possession ratio (MPR) }=92.3 \% \text { (assumption). } \\
\text { From data on file, Sanofi Genzyme, December 31, 2015. } \\
\text { From unpublished claims analysis, DataMart Multiplan (IMPACT) database } \\
\text { licensed from OPTUM Insight Life Science by Sanofi US Services, 2013. } \\
\text { cap = capsules; ERT = enzyme replacement therapy; SRT = substrate reduction } \\
\text { therapy; U = unit. }\end{array}$} \\
\hline
\end{tabular}

data indicating otherwise), and we assumed that an MPR less than $100 \%$ had no effect on the efficacy or safety of the treatment. As with ERT, we assumed no discontinuation for SRT.

\section{Analyses}

In the base-case analysis, we used the input values presented in Table 1, Table 2, and Table 3 to calculate the annual and 3-year cumulative pharmaceutical, administration, and total costs for the population, as well as the PMPM cost for the current and new markets. For the new market, the eliglustat uptake was drawn equally from imiglucerase and velaglucerase, while the sites of care distribution remained the same. For the sensitivity analysis, the 3-year cumulative budgets for the current and new markets were calculated and compared. The following parameters were varied: the average weight of patients was increased by $20 \%$ (from $72.0 \mathrm{~kg}$ to $86.4 \mathrm{~kg}$ ); full adherence was assumed in terms of the MPR (from 92.3\% to 100\%); and the number of infusions per year was increased from 24 to 26 .

As payer trends have supported an increased focus on site of care management and have shifted away from hospital-based infusions, ${ }^{20} 3$ new formularies based on site of care were evaluated.

1. Scenario 1: Maturing shift to eliglustat. Eliglustat's market share was increased in the new market and uptake was drawn from the hospital setting rather than evenly from all sites. In the current market, market shares for eliglustat and all other therapies remained unchanged from the base case. 
TABLE 4 Results of Base-Case Analysis

\begin{tabular}{|c|c|c|c|c|}
\hline Costs, $\$ \mathrm{a}$ & Current Market & New Market & Difference & Change, $\%$ \\
\hline \multicolumn{5}{|l|}{ Annual budget } \\
\hline Pharmaceutical & $11,078,520$ & $9,602,271$ & $-1,476,248$ & -13.3 \\
\hline Administration & 132,461 & 82,000 & $-50,461$ & -38.1 \\
\hline Total, overall population & $11,210,981$ & $9,684,271$ & $-1,526,710$ & -13.6 \\
\hline Total, PMPM & 0.187 & 0.161 & -0.025 & -13.6 \\
\hline \multicolumn{5}{|c|}{ Three-year cumulative budget } \\
\hline Pharmaceutical & $33,235,559$ & $28,806,813$ & $-4,428,745$ & -13.3 \\
\hline Administration & 397,384 & 246,000 & $-151,384$ & -38.1 \\
\hline Total, overall population & $33,632,943$ & $29,052,813$ & $-4,580,130$ & -13.6 \\
\hline
\end{tabular}

Note: Since all inputs remained the same in each year of the model time horizon, the annual budget was the same for years 1, 2, and 3 .

a All costs are expressed in 2017 U.S. dollars.

PMPM = per member per month.

Shifts of $50 \%$ and $100 \%$ of patients receiving ERT infusions in the hospital outpatient setting to eliglustat were tested, and the cost per vial used was recalculated based on the revised proportions in each site. This scenario evaluates the effect of shifting patients from ERT infusion in a hospital setting (most costly) to eliglustat.

2. Scenario 2: Site of care management. The proportion of patients receiving hospital infusions for ERTs was substantially reduced such that the site of care distribution for both current and new markets was changed from $36 \%, 32 \%$, and $32 \%$ to $58 \%, 32 \%$, and $10 \%$ for home, outpatient infusion center, and outpatient hospital setting, respectively. In this scenario, eliglustat's market share was unchanged from the base case. This scenario reflects redirection strategies that aim to manage the cost of ERT infusions by redirecting patients from hospital-based infusions to infusions at the patient's home.

3. Scenario 3: Conservative markup. For the current and new markets, the ERT cost markup was 20\% at all sites of care, rather than 20\%,25\%, and 100\% for home, outpatient infusion clinic, and outpatient hospital respectively. The last 2 scenarios assume a conservative estimation of the cost of infusions.

In all analyses, costs remained constant throughout the 3 -year time horizon with no inflation or discounting as budget holders are generally interested in the expected effect at a point in time without discounted cost. ${ }^{24}$

\section{Results}

\section{Base-Case Analysis}

In the base-case analysis, when uptake was drawn equally from all sites of care, increased use of eliglustat in the new market resulted in an annual savings of \$1,526,710 (13.6\%) compared with the current market (Table 4). Over a 3-year time horizon, the total savings was $\$ 4,580,130$. Most of the cost savings was attributable to reductions in pharmaceutical costs (reduction of $\$ 4,428,745)$. The reduction in administration costs was $\$ 151,384$. The annual PMPM cost saving was $\$ 0.025$.

\section{Sensitivity Analyses}

In the sensitivity analysis, an increase in patients' weight from $72 \mathrm{~kg}$ to $86 \mathrm{~kg}$ or an increase in the ERT dose from $47.4 \mathrm{U} / \mathrm{kg}$ to $56.9 \mathrm{U} / \mathrm{kg}$ resulted in a 3-year cumulative budget increase for both markets (an added $\$ 6,529,022$ for the current market and $\$ 4,016,553$ for the new market; Table 5). For both cases, the new market compared to the current market resulted in a savings of $\$ 7,092,599$ (17.7\%). Similarly, when patient adherence improved, the 3-year cumulative budget increased for both markets (an added $\$ 2,912,298$ for the current market and \$2,489,729 for the new market), and the new market compared to the current market resulted in a savings of $\$ 5,002,699$ (13.7\%).

\section{Scenario Analysis}

As shown in Table 5, when $50 \%$ of patients receiving infusions in the hospital outpatient setting were shifted to eliglustat for Scenario 1, the 3-year cumulative savings increased from $\$ 4,580,130$ in the base case to $\$ 5,162,841$ (15.4\%) and PMPM savings increased to $\$ 0.029$. The reweighted site of care distribution calculated the costs per vial used in the analysis as $\$ 2,261, \$ 1,963$, and $\$ 1,111$ for imiglucerase $400 \mathrm{U}$, velaglucerase $400 \mathrm{U}$, and taliglucerase $200 \mathrm{U}$, respectively. When $100 \%$ of patients receiving infusions in the hospital outpatient setting were shifted to eliglustat, the 3-year cumulative savings increased further to $\$ 7,700,456$ (22.9\%), and PMPM savings increased to $\$ 0.043$. The reweighted site of care distribution calculated the costs per vial used in the analysis to be $\$ 1,940$, $\$ 1,684$, and $\$ 953$ for imiglucerase $400 \mathrm{U}$, velaglucerase $400 \mathrm{U}$, and taliglucerase $200 \mathrm{U}$, respectively.

In Scenario 2, when the proportion of patients receiving infusions in hospital was substantially reduced, such that the site of care distribution for both current and new markets was 
TABLE 5 Results of the Sensitivity and Scenario Analyses

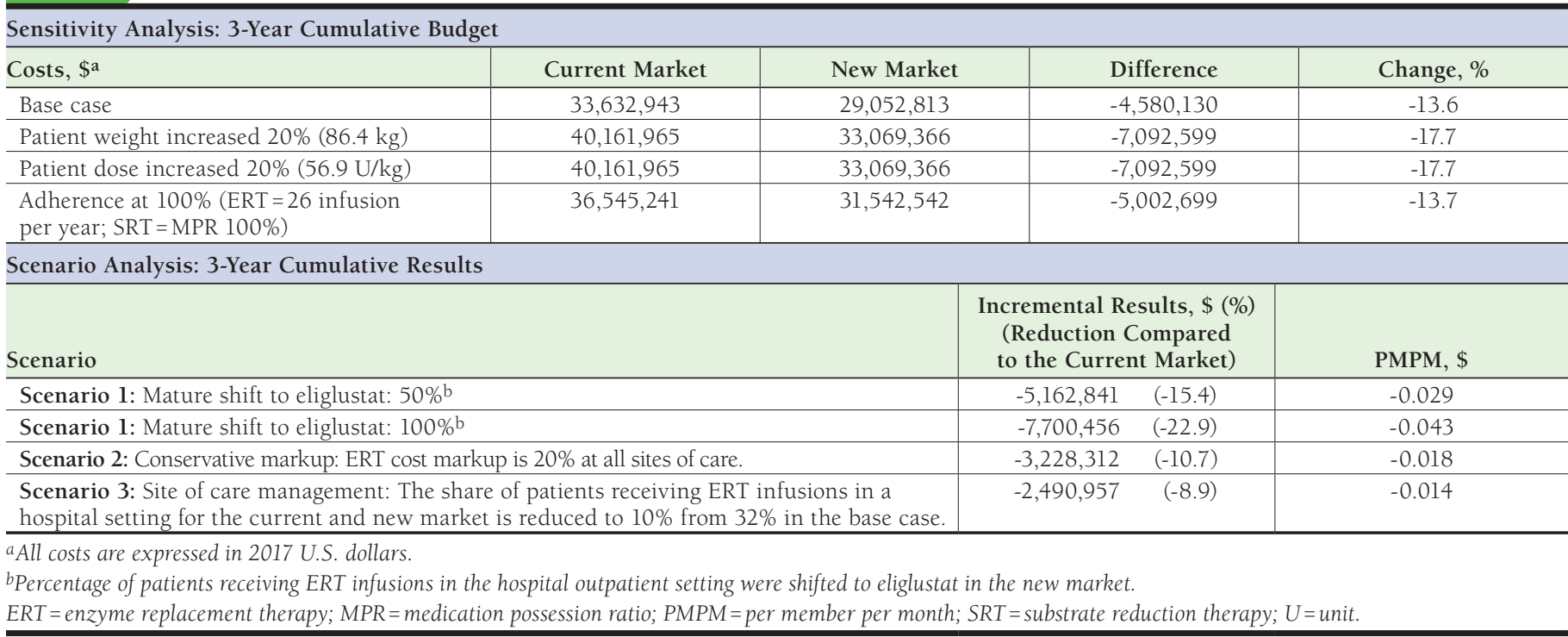

$58 \%, 32 \%$, and $10 \%$ for home, outpatient clinic, and hospital outpatient, respectively, the 3 -year cumulative savings comparing the new market to the current market were $\$ 2,490,957$ (8.9\%), and the PMPM savings were $\$ 0.014$, indicating that greater utilization of eliglustat was cost saving.

In Scenario 3, when the pharmaceutical markup was 20\% at all 3 sites of care, there was a 3-year cumulative savings of $\$ 3,228,312(10.7 \%)$, and PMPM savings of $\$ 0.018$.

\section{Discussion}

The results of this budget impact analysis indicate that increased use of eliglustat would result in significant cost savings to third-party payers in the United States. This is largely the result of shifting patients from more costly infusion-based therapy, particularly outpatient hospital infusions, to oral eliglustat. The greatest savings occurred when patients' weight or ERT drug dosage was increased by 20\% (17.7\% savings), followed by when $100 \%$ and $50 \%$ of the uptake for eliglustat was taken from the outpatient hospital rather than evenly from all sites (22.9\% and $15.4 \%$ savings, respectively).

When we tested more conservative scenarios, in which it was assumed that the overall cost of infusions was reduced, we found that increasing the use of eliglustat was still cost saving.

The results of our analysis are not surprising considering the known high cost of infusion. Payer trends have recently supported increased focus on site of care management and shifts away from hospital-based infusions. Site of care redirection programs have shown to be effective at managing the costs associated with ERT infusions. ${ }^{25}$ However, results from this analysis indicate that even when patients were redistributed across sites with a sizable proportion allocated to a home-based site of care (least expensive), increased use of eliglustat still resulted in meaningful cost savings. The results suggest that cost savings are also likely achievable from starting patients on eliglustat from the outset of treatment instead of infusion.

Switching from infusion to oral therapy, as well as bypassing infusions in favor of oral therapy, is not new. Oral therapy is often the favored route of administration so long as there is similar bioavailability, little therapeutic difference, and patients are clinically stable enough to manage oral therapy. ${ }^{26}$ Common drug classes involved in IV to oral administration include diuretics, corticosteroids, analgesics, antivirals, cardiovascular agents, antifungals, gastrointestinal agents, and antibacterials. ${ }^{27}$ The benefits of oral therapy compared with those of infusion include reduced cannula-related infections, reduced incidences of thrombophlebitis, and cost savings. ${ }^{26}$

In addition, patients can benefit from the convenience of an orally administered drug taken at home. In the phase 3 trial of eliglustat, an informal questionnaire was completed by all patients at screening and 12 months after treatment initiation for the eliglustat group. Ninety-four percent of patients in both treatment groups (eliglustat and imiglucerase) expressed a preference for oral treatment at the time of screening, and $93 \%$ of those who were taking eliglustat confirmed their preference for oral treatment after 12 months. ${ }^{13}$ Reasons stated for their preference for oral treatment included convenience, ability to take at home, feeling better after treatment, and fewer side effects. ${ }^{13}$

\section{Limitations}

This study has some limitations to consider. First, the generalizability to all insurance plans is limited, given that there are large variations in plan sizes and in the prevalence of GDl by region in the United States. The inputs used in this analysis, including plan size and number of eligible patients, 
market shares, distribution of patients across sites of care, and associated pharmaceutical cost markups, reflect a typical large regional health plan. We assumed payers would be responsible for both pharmacy and administration costs.

Second, the model was developed for cost comparison only and takes no position with respect to product safety, efficacy, or outcomes. Entering less than 100\% for the MPR was assumed to affect only overall drug costs and not efficacy or safety. Third, we also did not consider copayment and coinsurance, as this would introduce significant complexity related to shifting between medical and pharmacy benefits due to changes in care locations and routes of administration, yet have minimal effect on the analysis.

Finally, these analyses assume a dosage of $84 \mathrm{mg}$ of eliglustat twice daily, which is the recommended dose for patients who are extensive and intermediate metabolizers, and we did not account for the proportion of patients who are poor metabolizers (approximately 5\%-10\%) who have a reduced recommended dosage of $84 \mathrm{mg}$ once daily. It is possible that cost savings could be greater by accounting for poor metabolizers, although it is difficult to substantiate without further analysis.

\section{Conclusions}

Based on these analyses, increased use of eliglustat resulted in meaningful cost savings to a payer's overall budget. Third-party payers can potentially save money if patients switch from ERT infusion to orally administered eliglustat. Cost savings are also likely achievable from starting patients on eliglustat from the outset of treatment instead of starting with infusion therapy.

\section{Authors}

LUBA NALYSNYK MD, MPH, Sanofi Genzyme, Cambridge, Massachusetts. REBECCA SUGARMAN, MS; CLIFFORD CELE, BA; and ALEXANDRA WARD, PhD, MRPharmS, Evidera, Waltham, Massachusetts. JENNIFER UYEI, PhD, MPH, Evidera, San Francisco, California.

AUTHOR CORRESPONDENCE: Alexandra Ward, PhD, MRPharmS, Evidera, 500 Totten Pond Rd., Fifth Fl., Waltham, MA 02154. Tel.: 781.960.0227; E-mail: alex.ward@evidera.com.
This study was presented as a poster at the Academy of Managed Care Pharmacy Nexus 2016, October 3-6, 2016; National City, MD, and at the International Society for Pharmacoeconomics and Outcomes Research, 22nd Annual International Meeting; May 20-24, 2017; Boston, MA.

\section{ACKNOWLEDGMENTS}

The authors thank Janet Dooley of Evidera's Editorial and Design Services team for her assistance with the editing and preparation of this manuscript.

\section{REFERENCES}

1. Bennett LL, Turcotte K. Eliglustat tartrate for the treatment of adults with type 1 Gaucher disease. Drug Des Devel Ther. 2015;9:4639-47.

2. Nalysnyk L, Rotella P, Simeone JC, Hamed A, Weinreb N. Gaucher disease epidemiology and natural history: a comprehensive review of the literature. Hematology. 2017;22(2):65-73.

3. Bennett LL, Mohan D. Gaucher disease and its treatment options. Ann Pharmacother. 2013;47(9):1182-93.

4. Elstein D, Burrow TA, Charrow J, et al. Home infusion of intravenous velaglucerase alfa: experience from pooled clinical studies in 104 patients with type 1 Gaucher disease. Mol Genet Metab. 2017;120(1-2):111-15.

5. Kaplan P, Andersson HC, Kacena KA, Yee JD. The clinical and demographic characteristics of nonneuronopathic Gaucher disease in 887 children at diagnosis. Arch of Pediatr Adolesc Med. 2006;160(6):603-08.

6. Balwani M, Burrow TA, Charrow J, et al. Recommendations for the use of eliglustat in the treatment of adults with Gaucher disease type 1 in the United States. Mol Genet Metab. 2016;117(2):95-103.

7. Pastores GM, Weinreb NJ, Aerts H, et al. Therapeutic goals in the treatment of Gaucher disease. Semin Hematol. 2004:41(4 Suppl 5):4-14.

8. Ficicioglu C. Review of miglustat for clinical management in Gaucher disease type 1. Ther Clin Risk Manag. 2008;4(2):425-31.

9. Burrow TA, Grabowski GA. Velaglucerase alfa in the treatment of Gaucher disease type 1. Clin Investig (Lond). 2011;1(2):285-93.

10. Belmatoug N, Di Rocco M, Fraga C, et al. Management and monitoring recommendations for the use of eliglustat in adults with type 1 Gaucher disease in Europe. Eur J Intern Med. 2017;37:25-32.

11. Lukina E, Watman N, Dragosky M, et al. Eliglustat, an investigational oral therapy for Gaucher disease type 1: phase 2 trial results after 4 years of treatment. Blood Cells Mol Dis. 2014;53(4):274-76.

12. Lukina E, Watman N, Arreguin EA, et al. Improvement in hematological, visceral, and skeletal manifestations of Gaucher disease type 1 with oral eliglustat tartrate (Genz-112638) treatment: 2-year results of a phase 2 study. Blood. 2010;116(20):4095-98

13. Cox TM, Drelichman G, Cravo R, et al. Eliglustat compared with imiglucerase in patients with Gaucher's disease type 1 stabilised on enzyme replacement therapy: a phase 3, randomised, open-label, non-inferiority trial. Lancet. 2015;385(9985):2355-62.

14. Mistry PK, Lukina E, Ben Turkia H, et al. Effect of oral eliglustat on splenomegaly in patients with Gaucher disease type 1: the ENGAGE randomized clinical trial. JAMA. 2015;313(7):695-706

15. Mistry PK, Lukina E, Ben Turkia H, et al. Outcomes after 18 months of eliglustat therapy in treatment-naive adults with Gaucher disease type 1: the phase 3 ENGAGE trial. Am J Hematol. 2017;92(11):1170-76.

16. Cox TM, Drelichman G, Cravo R, et al. Eliglustat maintains long-term clinical stability in patients with Gaucher disease type 1 stabilized on enzyme therapy. Blood. 2017;129(17):2375-83.

17. Hicks JK, Swen JJ, Thorn CF, et al. Clinical Pharmacogenetics Implementation Consortium guideline for CYP2D6 and CYP2C19 genotypes and dosing of tricyclic antidepressants. Clin Pharmacol and Ther. 2013;93(5):402-08 
18. Peterschmitt MJ, Cox GF, Ibrahim J, et al. A pooled analysis of adverse events in 393 adults with Gaucher disease type 1 from four clinical trials of oral eliglustat: evaluation of frequency, timing, and duration. Blood Cells $\mathrm{Mol}$ Dis. 2018;68:185-91

19. Einodshofer MT, Duren LN. Cost management through care management, part 2: the importance of managing specialty drug utilization in the medical benefit. Am Health Drug Benefits. 2012;5(6):359-64.

20. Polinski JM, Kowal MK, Gagnon M, Brennan TA, Shrank WH. Home infusion: safe, clinically effective, patient preferred, and cost saving. Healthc (Amst). 2017;5(1-2):68-80

21. Milligan A, Hughes D, Goodwin S, Richfield L, Mehta A. Intravenous enzyme replacement therapy: better in home or hospital? Br J Nurs. 2006;15(6):330-33

22. Truven Health Analytics, IBM Watson Health. Micromedex. RED BOOK Online. 2017. Database. Available at: https://ruvenhealth.com/Products/ Micromedex/Product-Suites/Clinical-Knowledge/RED-BOOK. Accessed August 10, 2018.

23. Einodshofer MT, Ellis M. The challenge of specialty pharmacy: mitigating costs while providing quality care. Special Pharmacy Times. August 8, 2013. Available at: https://www.specialtypharmacytimes.com/publications/ specialty-pharmacy-times/2013/july_august-2013/the-challenge-of-specialtypharmacy-mitigating-costs-while-providing-quality-care. Accessed July 22, 2018

24. Sullivan SD, Mauskopf JA, Augustovski F, et al. Budget impact analysisprinciples of good practice: report of the ISPOR 2012 budget impact analysis good practice II task force. Value Health. 2014;17(1):5-14.
25. Chan W, Spykerman T, Cummings M, et al. Results from a site of care redirection program targeting members on enzyme replacement therapy. J Manag Care Pharm. 2013;19(8):683 [Abstract]. Available at: https://www. jmcp.org/doi/pdf/10.18553/jmcp.2013.19.issue-8.

26. Cyriac JM, James E. Switch over from intravenous to oral therapy: a concise overview. J Pharmacol Pharmacother. 2014;5(2):83-87.

27. Banko H, Goldwater SH, Adams E. Smoothing the path for intravenous (IV) to oral (PO) conversion: where have we come in 11 years? Hosp Pharm. 2009;44(11):959-67.

28. Cerdelga (eliglustat) capsules for oral use. Genzyme Ireland. August 2014. Available at: https://www.accessdata.fda.gov/drugsatfda_docs/ label/2014/205494Origls000lbl.pdf. Accessed August 20, 2018.

29. Zavesca (miglustat) capsules, for oral use. Actelion Pharmaceuticals US. 2014. Available at: https://www.accessdata.fda.gov/drugsatfda_docs/ label/2014/021348s010lbl.pdf. Accessed August 20, 2018.

30. Cerezyme (imiglucerase for injection). Genzyme. May 2011. Available at: https://www.cerezyme.com/ /media/CerezymeUS/Files/pdf/cerezyme_ pi.pdf. Accessed August 20, 2018.

31. Vpriv (velaglucerase alfa for injection) for intravenous use. Shire Human Genetic Therapies. 2013. Available at: https://www.accessdata.fda.gov/drugsatfda_docs/label/2013/022575s013lbl.pdf. Accessed August 20, 2018.

32. Elelyso (taliglucerase alfa) for injection, for intravenous use. Pfizer Labs. 2012. Available at: https://www.accessdata.fda.gov/drugsatfda_docs/ label/2012/022458lbl.pdf. Accessed August 20, 2018. 